\title{
Glucagon-like peptide-I receptor agonists as add-on therapy to basal insulin in patients with type 2 diabetes: a systematic review
}

This article was published in the following Dove Press journal:

Diabetes, Metabolic Syndrome and Obesity:Targets and Therapy 2 July 2012

Number of times this article has been viewed

\section{Helen Berlie \\ Kathryn M Hurren \\ Nicole R Pinelli}

Eugene Applebaum College of Pharmacy and Health Sciences, Wayne State University, Detroit, MI, USA
Correspondence: Nicole R Pinelli Department of Pharmacy Practice, Eugene Applebaum College of Pharmacy and Health Sciences, Wayne State University, 259 Mack Avenue, Detroit, MI 4820I, USA

Tel +I 3139937555

Fax + I 3135775369

Email nickipinelli@wayne.edu
Abstract: The prevalence of obesity and diabetes continues to rise in the US. Glucagon-like peptide-1 receptor agonist (GLP-1RA) is an effective treatment option for type 2 diabetes mellitus (T2DM) that promotes weight loss. Common and effective treatment options added to metformin therapy (basal insulin, sulfonylureas, and pioglitazone) contribute to weight gain, which makes the addition of GLP-1RAs advantageous. Exenatide was the first agent in this class and has recently been approved for use in combination with insulin glargine by the US Food and Drug Administration and the European Medicines Agency. Until recently, there was a lack of data examining basal insulin combined with these agents. The main purpose of this article is to review the prospective interventional data on the safety and efficacy of GLP-1RAs (exenatide, liraglutide, albiglutide, lixisenatide) combined with basal insulin therapy in nonpregnant adults with T2DM. Databases searched were PubMed, Cochrane Central Register of Controlled Trials and the Database of Systematic Reviews (inception to January 2012). Abstracts presented at relevant diabetes and endocrine meetings from 2009 to 2011 were also reviewed, as were reference lists of identified publications. A total of five studies met the criteria and were included in the review. Data from these studies demonstrated that this combination therapy offers advantages for the treatment of diabetes, such as additional lowering of A1c without major risk for hypoglycemia, lower basal insulin requirements, decreased postprandial glucose levels (with or without fasting plasma glucose decreases), and weight loss, or at the very least, less weight gain. However, the gastrointestinal side effects and high cost of these agents may limit their use. This review demonstrates that adding a GLP-1RA to an existing basal insulin regimen is a reasonable treatment strategy in nonpregnant adult patients with T2DM.

Keywords: exenatide, liraglutide, lixisenatide, GLP-1, type 2 diabetes

\section{Introduction}

The tandem obesity and diabetes epidemics are major public health problems, with $34 \%$ and $11.3 \%$ of adults in the US affected, respectively. ${ }^{1,2}$ Although glycemic control and weight loss have been shown to improve outcomes for patients with type 2 diabetes (T2DM), the achievement and maintenance of these goals remains a significant challenge for the majority of patients. ${ }^{3-5}$ The American Diabetes Association and the European Association for the Study of Diabetes recommend metformin be prescribed initially for all patients without contraindications. ${ }^{6}$ In most cases, basal insulin is eventually necessary to achieve glycemic control. There are several adjunctive antihyperglycemic therapies recommended for individuals unable to achieve adequate glycemic control on basal insulin alone. Therapeutic options to add to metformin and basal insulin include bolus insulin or a thiazolidinedione, which can contribute 
to further weight gain, dipeptidyl peptidase IV (DPP-IV) inhibitor, which is associated with weight neutrality, or a glucagon-like peptide-1 receptor agonist (GLP-1RA), which produces benefit in both glycemia and weight loss.

GLP-1 is an endogenous hormone secreted from L cells of the small intestine upon ingestion of meals. Interacting with GLP-1 receptors, it stimulates glucose-dependent insulin secretion, suppresses postprandial glucagon release from pancreatic alpha cells, signals satiety, and slows gastric emptying. ${ }^{7-10}$ Endogenous GLP-1 has a short half-life of 1-2 minutes and a diminished effect in patients with T2DM. ${ }^{11}$ Thus, exogenously administered GLP-1RAs have a therapeutic role in restoring beneficial effects to decrease hemoglobin A1c by approximately $1 \%$ and promote weight loss of $2-3 \mathrm{~kg}$ over six months. ${ }^{12}$ The first agent of this class, exenatide, is administered subcutaneously twice daily within 60 minutes prior to meals, and mainly targets postprandial blood glucose (PPG). ${ }^{13,14}$ Liraglutide was approved in 2010 and has a longer half-life amenable to once-daily dosing, with greatest effect on fasting plasma glucose (FPG). ${ }^{14,15}$

Recently extended exenatide (weekly administration) has been approved for the management of T2DM. Several additional long-acting GLP-1RAs are currently in clinical development, including albiglutide (weekly administration) and lixisenatide (daily administration). These agents have a low risk of hypoglycemia, as monotherapy and most common side effects are nausea, vomiting and diarrhea.

Recently, exenatide was approved by the US Food and Drug Administration (FDA) and the European Medicines Agency for use in combination with insulin glargine, with or without concomitant metformin or thiazolidinedione. The combination of GLP-1RA and insulin has several potential advantages, including decreased exogenous insulin requirement, weight maintenance or loss, and targeting both fasting and postprandial hyperglycemia. Potential concerns include increased risk of hypoglycemia and other adverse effects, as well as cost. Previous articles have reviewed retrospective and observational studies of GLP-1RA and insulin combination therapy. The purpose of this article is to review prospective interventional data on the safety and efficacy of GLP-1RA and basal insulin combination therapy in nonpregnant adult patients with T2DM.

\section{Methods}

\section{Search strategy}

Three independent reviewers queried PubMed and the Cochrane Central Register of Controlled Trials and Database of Systematic Reviews (inception to January 2012), combining the search terms GLP-1RA (exenatide, liraglutide, albiglutide, lixisenatide) and insulin. The search results were limited to those published in English and conducted in adult humans. Abstracts from the annual meetings of the American Diabetes Association, American Association of Clinical Endocrinologists, and European Association for the Study of Diabetes from 2009 through 2011 were queried using the same search terms. Reference lists of identified publications were also reviewed to identify any additional trials.

\section{Eligibility criteria}

Publications were included if they (1) were clinical trials, (2) included nonpregnant adult subjects with T2DM, (3) examined the efficacy and safety of therapeutic-dose GLP-1RA (exenatide, liraglutide, extended exenatide, albiglutide, or lixisenatide) in combination with basal insulin and controlled with basal insulin, and (4) included measures of glycemic efficacy as an outcome. Studies were excluded if they were published in a language other than English. Studies including the experimental GLP-1RA, taspoglutide, were also excluded due to discontinuation of its clinical development. Abstracts were excluded if they reported results of an included publication. Extension studies and post hoc analyses were also excluded.

\section{Study selection}

Three reviewers independently screened all abstracts generated by the search for inclusion and exclusion criteria. Full-text potentially relevant articles were reviewed independently to determine eligibility. Discrepancies between the reviewers were to be resolved by consensus; however, no discrepancies arose.

\section{Data extraction}

A structured electronic data-extraction file was developed for compiling information from selected studies. Study design, sample size, study duration, interventions, risk of bias measures, and population characteristics including age, sex, race, duration of diabetes, baseline A1c, FPG, and body mass index (BMI) were recorded. For glycemic efficacy, we extracted the mean change in A1c, FPG, and PPG from baseline to study end point, the proportion of participants achieving A1c target goals, and change in insulin dose. Cardiovascular risk-factor end points extracted included the mean change from baseline in body weight, lipids (triglycerides, total cholesterol, low-density lipoprotein [LDL] and high-density lipoprotein [HDL]), and systolic and diastolic blood pressure. We extracted data on the proportion of patients per treatment 
group who experienced hypoglycemia. Occurrence of gastrointestinal and other serious adverse events was also extracted. An independent reviewer examined the accuracy of the extracted data. Data were converted to International Systems of Units symbols where appropriate. Data are expressed as mean \pm standard deviation or percentage, unless otherwise noted, for continuous and categorical outcomes, respectively.

\section{Risk of bias}

To assess the included publications for potential selection, performance, attrition, detection, and reporting biases, we evaluated whether the investigators described sequence generation, concealment of allocation, and blinding. We also reviewed the included publications for incomplete outcome data, selective outcome reporting, and other sources of potential bias.

\section{Results}

\section{Study characteristics}

The search strategy employed is depicted in Figure 1 in accordance with the Preferred Reporting Items for Systematic Reviews and Meta-Analyses guidelines. ${ }^{16}$ A total of 883 exenatide, 312 liraglutide, 15 lixisenatide, and 14 albiglutide publications were identified. After critical evaluation, five publications met all of the eligibility criteria for the systematic review. A summary of the study design, population characteristics, and interventions of the publications meeting the eligibility criteria for the systematic review are shown in Table 1. No studies of extended exenatide or albiglutide met the eligibility criteria.

\section{Risk of bias}

Methods used to generate the sequence of randomization and concealment of allocation were adequately described in $20 \%$ $(\mathrm{n}=1)$ and $0 \%(\mathrm{n}=0)$ of the included trials, respectively. The majority of studies had a blinded trial design $(n=60 \%)$. Incomplete and selective outcome reporting were present in $0 \%(n=0)$ and $20 \%(n=1)$ of the included trials, respectively. Pharmaceutical manufacturers sponsored at least $60 \%$ $(n=3)$ of the included trials (two abstracts did not report funding source).

\section{Clinical trials}

\section{Exenatide}

The major study behind the FDA approval for the use of exenatide as an adjunct to insulin glargine was conducted by Buse et al. ${ }^{17}$ This was a randomized, double-blind, placebo-controlled, parallel group, multicenter, 30-week trial (Table 1). Patients with age at least 18 years, T2DM (A1c $7.1 \%-10.5 \%)$, BMI $\leq 45 \mathrm{~kg} / \mathrm{m}^{2}$ and stable weight ( $<5 \%$ weight change in body weight over 3 months) who

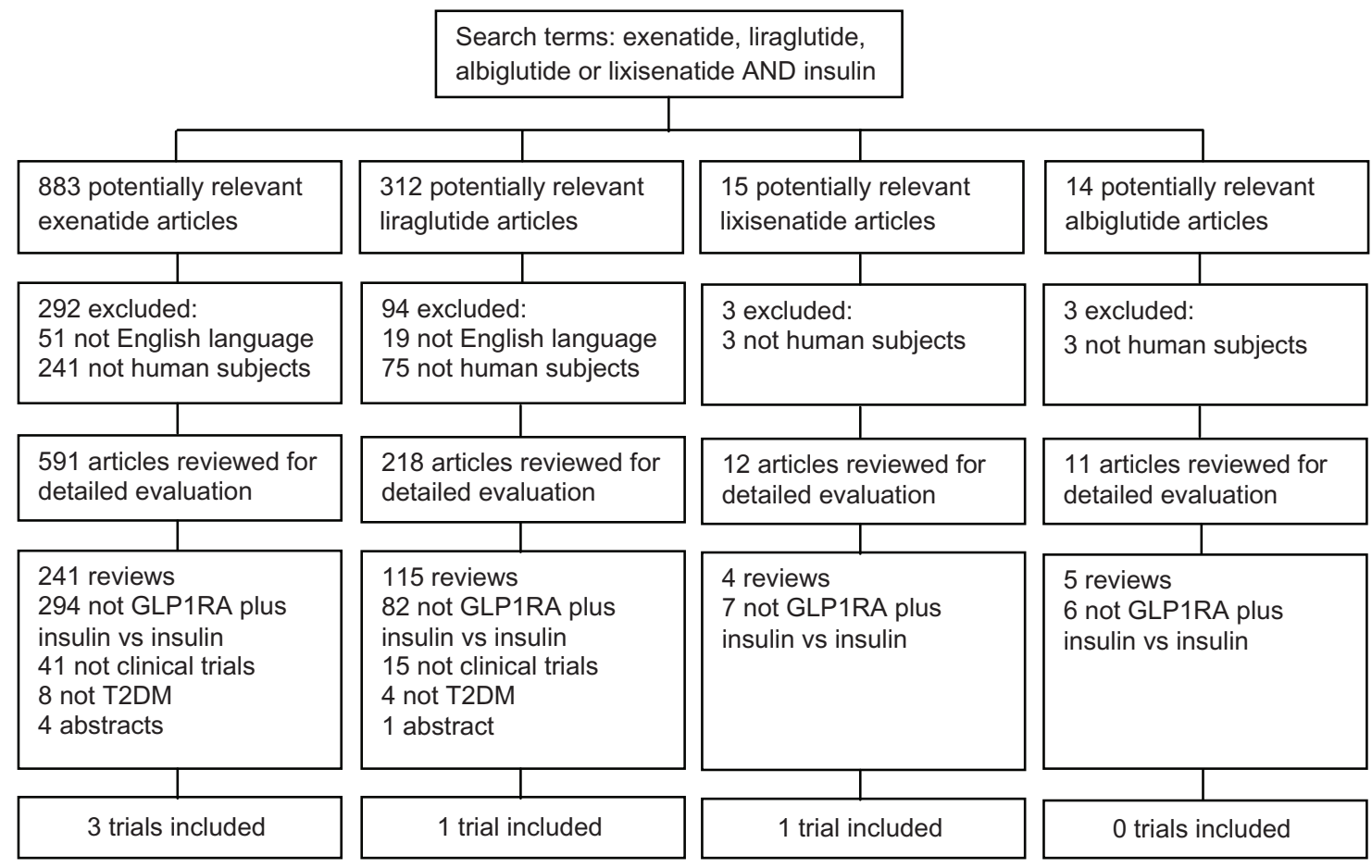

Figure I Search strategy. 


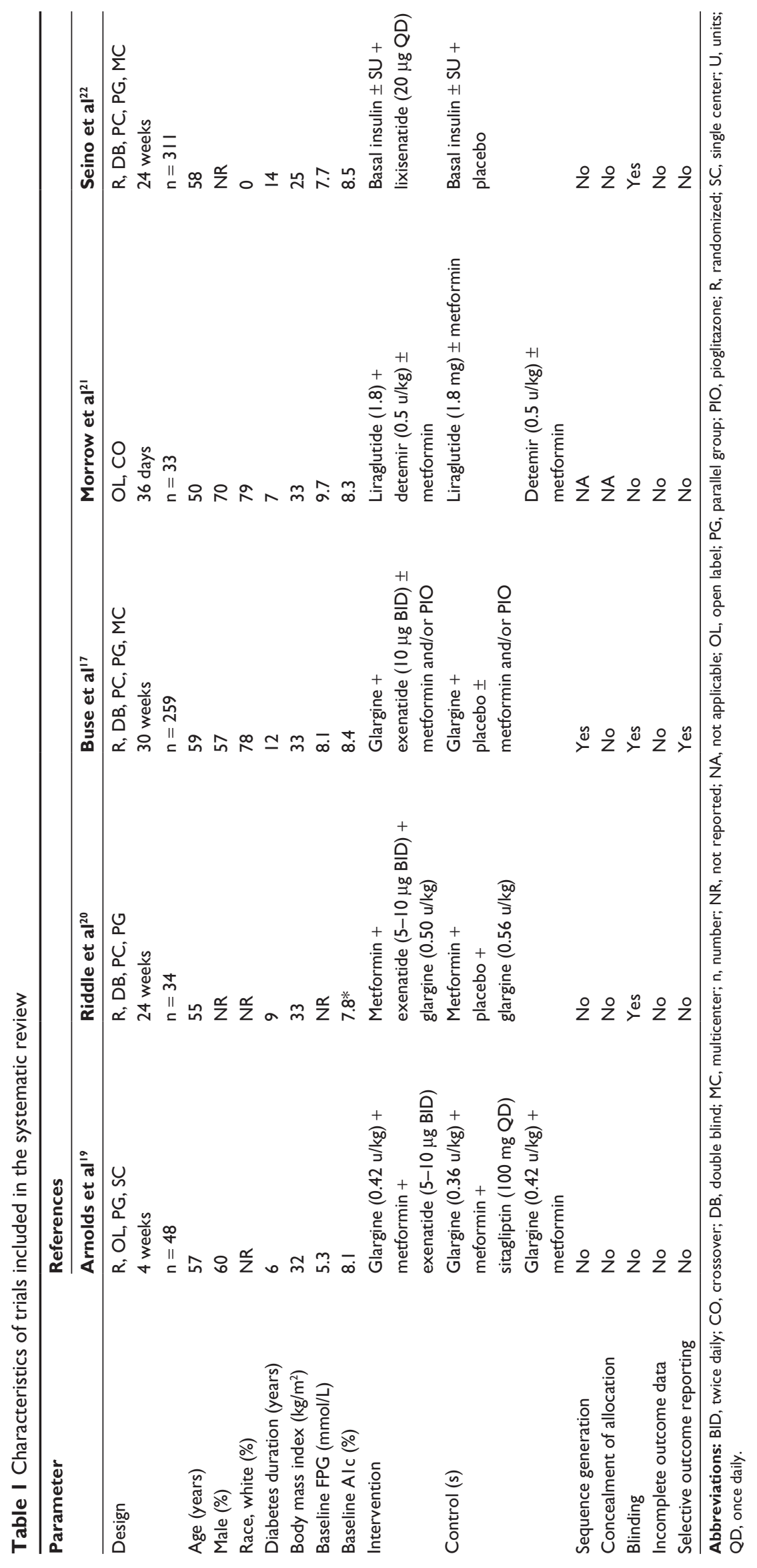


were treated with insulin glargine (minimum of 20 units per day) with or without metformin and/or pioglitazone for at least 3 months were included in this study. The primary end point was change in A1c from baseline at 30 weeks.

A total of 259 participants were randomized and received exenatide titrated to $10 \mathrm{mcg}$ twice daily $(\mathrm{n}=137)$ or matching placebo $(\mathrm{n}=122)$ injections. Glargine doses were prospectively reduced by $20 \%$ in those with a baseline A1c $\leq 8 \%$. After 5 weeks, all study participants used the treat-to-target algorithm to adjust glargine to achieve fasting glucose of $<5.6 \mathrm{mmol} / \mathrm{L} .{ }^{18}$ Baseline characteristics between exenatide and placebo groups were similar, with the exceptions of sex (females $49 \%$ vs $36 \%$, respectively) and use of prestudy oral antihyperglycemic agents (metformin $66 \%$ vs $75 \%$; pioglitazone $2 \%$ vs $5 \%$; both $17 \%$ vs $7 \%$, respectively).

At the end of the study, A1c was reduced to a greater extent in the exenatide group than in the placebo group ( $-1.74 \%$ [95\% CI $-1.91 \%$ to $-1.56 \%$ ] vs $-1.04 \%$ [ $-1.22 \%$ to $-0.86 \%]$; between-group difference, $-0.69 \%[-0.93 \%$ to $-0.46 \%$ ]; $P<0.001)$. Participants in the exenatide group achieved A1c $\leq 7.0 \%$ more frequently when compared to placebo (60\% [51\%-69\%] vs 35\% [25\%-45\%]; betweengroup difference, $25 \%$ [12\%-39\%]; $P<0.001)$. In addition, A1c $\leq 6.5 \%$ was achieved more often in the exenatide group over placebo (40\% [30\%-49\%] vs $12 \%$ [6\%-17\%]; betweengroup difference, 28\% [17\%-39\%]; $P<0.001)$. Insulin doses were increased to a greater extent in the placebo group compared to the exenatide group (20 units/day [16-24 units/ day] vs 13 units/day [9-17 units/day]; between-group difference, -6.5 units/day $[-12.3$ to -0.8 units/day $; P=0.03$ ). The decrease in FPG was similar for both the exenatide and placebo groups $(-1.6 \mathrm{mmol} / \mathrm{L}[-1.9$ to $-1.3 \mathrm{mmol} / \mathrm{L}]$ vs $-1.5 \mathrm{mmol} / \mathrm{L}[-1.8$ to $-1.2 \mathrm{mmol} / \mathrm{L}]$; between-group difference, $-0.1 \mathrm{mmol} / \mathrm{L}[-0.52$ to $0.32 \mathrm{mmol} / \mathrm{L}] ; P=0.63)$. Self-monitored blood glucose levels were lower with exenatide at the morning 2-hour postprandial time point (between-group difference, $-1.8 \mathrm{mmol} / \mathrm{L}[-2.5$ to $-1.2 \mathrm{mmol} / \mathrm{L}] ; P<0.001)$ and evening 2-hour postprandial time point (betweengroup difference, $-1.7 \mathrm{mmol} / \mathrm{L}[-2.3$ to $-1.1 \mathrm{mmol} / \mathrm{L}]$; $P<0.001$ ), but not at the midday 2 -hour postprandial time point (between-group difference, $-0.3 \mathrm{mmol} / \mathrm{L}[-0.8$ to $0.3 \mathrm{mmol} / \mathrm{L}] ; P=0.32$ ). Weight loss with exenatide was greater than that observed with placebo $(-1.8 \mathrm{~kg}[-2.5$ to $-1.1 \mathrm{~kg}$ ] vs $+1.0 \mathrm{~kg}$ [0.2 to $1.7 \mathrm{~kg}$ ]; between-group difference, $-2.7 \mathrm{~kg}[-3.7$ to $-1.7 \mathrm{~kg}] ; P<0.001)$. At 30 weeks, triglycerides, HDL, LDL, and non-HDL cholesterol did not differ between groups. The exenatide group demonstrated a greater decrease in systolic and diastolic blood pressures compared to placebo (between-group difference, $-4.4 \mathrm{mmHg}$ [-7.8 to $-1.0 \mathrm{mmHg}] ; P=0.01$ and $-3.4 \mathrm{mmHg}[-5.2$ to $-1.6 \mathrm{mmHg}$; $P<0.001$, respectively), but heart rate was increased (between-group difference, 3.0 beats/minute [0.8-5.2 beats/minute]; $P<0.01)$.

Hypoglycemic events per participant per year as well as minor hypoglycemic events did not significantly differ between groups $(P=0.49)$. Minor hypoglycemic episodes were defined as self-treated or self-limiting symptomatic episodes with a corresponding blood glucose level $<3 \mathrm{mmol} / \mathrm{L}$. No participants reported major hypoglycemic episodes in the exenatide group, compared to one participant in the placebo group. Major hypoglycemic episodes were defined as prompt recovery from loss of consciousness or seizure with the administration of glucagon or glucose, with a blood glucose level $<3 \mathrm{mmol} / \mathrm{L}$. Alternatively, a major episode could have been presumed if severe impairment in consciousness or behavior occurred and required third-party assistance. There was a higher withdrawal rate from the study for adverse events in the exenatide group compared to the placebo group ( $9 \%$ vs $1 \% ; P<0.01)$. The following adverse events occurred significantly more frequently with exenatide compared to placebo: nausea, diarrhea, vomiting, headache, and constipation.

Another study conducted by Arnolds et al examined the addition of exenatide or dipeptidyl-peptidase-4 (DPP-IV) inhibitor, sitagliptin, to existing therapy with glargine and metformin compared to continuation of glargine and metformin alone (Table 1). ${ }^{19}$ This was a randomized, parallel group, single-center, open-label, active-comparatorcontrolled, 4-week study. Participants were between the ages of 35 and 70 years, had duration of T2DM between 6 months and 10 years (A1c 7-10), and were treated with a stable regimen of metformin with or without a sulfonylurea, or a long or intermediate insulin (glargine, detemir, or NPH) with or without metformin. Additional criteria were BMI between 21.0 and $39.9 \mathrm{~kg} / \mathrm{m}^{2}$, a minimum of 3 months of stable therapy with antihypertensives or lipid-lowering agents, and lack of other clinically relevant medical conditions. The primary end point was the 6-hour postprandial blood glucose excursion following consumption of a standardized breakfast (618.2 kcal, $99.4 \mathrm{~g}$ of carbohydrates, $11.9 \mathrm{~g}$ of lipids, and $26.2 \mathrm{~g}$ of protein) after 4 weeks.

After a 4-8-week run-in period, during which insulin glargine was continued or initiated and titrated and sulfonylureas were discontinued, 48 participants were randomized to receive exenatide $(5 \mathrm{mcg}$ twice daily for 2 weeks, then 
10 mcg twice daily for 2 weeks $)+$ glargine + metformin $(\mathrm{n}=16)$, sitagliptin $(100 \mathrm{mg} /$ day $)+$ glargine + metformin $(n=16)$, or the control of glargine + metformin $(n=16)$. During the run-in and treatment periods, glargine doses were adjusted using a modified treat-to-target algorithm to achieve a goal FPG of $\leq 5.6 \mathrm{mmol} / \mathrm{L} .{ }^{18}$ Two days prior to the treatment phase, glargine doses for all participants were decreased by $20 \%$. Baseline A1c was reported as $7.9 \%$ for both the sitagliptin and control groups and $8.4 \%$ for the exenatide group; this difference was not reported to be statistically significant. No differences were reported among baseline demographics (Table 1).

At the end of the study, blood glucose excursions after the standardized meal were decreased to a greater extent, with both the exenatide and sitagliptin groups compared to the control group (between-group difference, $-17 \%[P=0.0036]$ and $-20 \%[P=0.0008]$, respectively). The difference between the exenatide and sitagliptin groups was not significant $(P=0.57)$. A1c levels were decreased in the exenatide, sitagliptin, and control groups $(-1.8 \%$ and $-1.5 \%$ vs $-1.2 \%$, respectively; $P=0.015$ for exenatide vs control). The target A1c level of $<7 \%$ was met by $80 \%$ of the exenatide group, $88 \%$ of the sitagliptin group, and $63 \%$ of the control group $(P<0.05$ for exenatide and sitagliptin groups compared to control). Insulin doses at baseline were similar to end-point doses in the exenatide (40.3 units/day [0.60 \pm 0.25 units/day] vs 41.1 units/day [0.42 \pm 0.18 units/day]) and sitagliptin groups (33.4 units/day [0.50 \pm 0.20 units/day] vs 35.0 units/day $[0.36 \pm 0.17$ units/day]), and were increased in the control group (32.3 units/day [0.50 \pm 0.21 units/day] vs 37.9 units/day $[0.42 \pm 0.20$ units/day]). End-of-treatment FBG levels were significantly decreased from baseline in both the exenatide (from 5.2 to $4.6 \mathrm{mmol} / \mathrm{L} ; P=0.0018$ ) and sitagliptin (from 5.3 to $4.7 \mathrm{mmol} / \mathrm{L} ; P=0.0016$ ) arms but not in the control group (from 5.2 to $4.9 \mathrm{mmol} / \mathrm{L} ; P=0.21$ ). Patients measured 7-point BG levels once a week and at the end of the study; these profiles were lower with both the exenatide and sitagliptin groups compared to the control group $(P<0.05$ for both). Body weight changed $-0.9 \pm 1.7 \mathrm{~kg}$ in the exenatide group, $0.1 \pm 1.6 \mathrm{~kg}$ in the sitagliptin group and $+0.4 \pm 1.5 \mathrm{~kg}$ in the control group ( $P=0.038$ for exenatide vs control). Significant decreases in total cholesterol were observed in the exenatide and sitagliptin groups when compared to control $(-0.24 \pm 0.48 \mathrm{mmol} / \mathrm{L}$ and $-0.27 \pm 0.61 \mathrm{mmol} / \mathrm{L}$ vs $0.30 \pm 0.52 \mathrm{mmol} / \mathrm{L}[P<0.01$ for both, respectively]). LDL cholesterol was also significantly decreased when compared to control in the exenatide and sitagliptin groups $(-0.30 \pm 0.46 \mathrm{mmol} / \mathrm{L}$ and $-0.28 \pm 0.42 \mathrm{mmol} / \mathrm{L}$ vs
$0.09 \pm 0.36 \mathrm{mmol} / \mathrm{L}[P<0.05$ for both $]$, respectively). No changes in HDL cholesterol were observed.

Minor hypoglycemia was experienced by five, two, and six subjects in the exantide, sitagliptin, and control groups, respectively. Minor episodes were defined as self-treatable episodes with or without blood glucose readings $<2.78 \mathrm{mmol} / \mathrm{L}$, or blood glucose levels $>2.78 \mathrm{mmol} / \mathrm{L}$ with symptoms only. Hypoglycemia occurred at rates of 10.1 events/person-year in the exenatide group, 3.3 events/person-year in the sitagliptin group, and 1.6 events/person-year in the control group. No major hypoglycemic episodes were reported in any group. Major episodes were defined as subjects not being able to treat the episode on their own. Gastrointestinal side effects accounted for $56.3 \%, 18.8 \%$, and $6.3 \%$ of adverse events experienced in the exenatide, sitagliptin, and control groups, respectively. There was one dropout in the exenatide group due to gastrointestinal side effects.

Riddle et al conducted a randomized, double-blind, placebo-controlled, parallel group, 24-week trial, which is available as an abstract (Table 1). ${ }^{20}$ Patients treated with metformin and one additional oral agent or insulin $(<0.4$ units $/ \mathrm{kg})$ were included in the study. During an 8-week run-in period, metformin was continued and the additional agent was switched to open-label exenatide 5-10 mcg twice daily. A total of 34 subjects were randomized to replace open-label exenatide with double-blind exenatide $(n=17)$ or placebo $(n=17)$. Insulin glargine was also initiated and titrated in all patients at randomization.

At the end of the 24 weeks, median A1c was $6.45 \%$ $(5.7 \%-9.3 \%)$ in the exenatide group and $7.30 \%(5.7 \%-9.8 \%)$ in the placebo group $(P=0.06)$. More participants in the exenatide group achieved A1c $<7.0 \%$ and $<6.5 \%$ compared to placebo $(76.5 \%$ vs $23.5 \% ; P=0.003$; and $47.1 \%$ vs $11.8 \% ; P=0.03$, respectively). The average insulin glargine dose in the exenatide group was $0.50 \pm 0.33$ units $/ \mathrm{kg} /$ day and $0.56 \pm 0.29$ units $/ \mathrm{kg} /$ day in the placebo group. Median difference in FPG was not significantly different between groups. Weight gain was observed to a lesser extent in the exenatide group versus the placebo group $(+0.4 \pm 1.1 \mathrm{vs}+4.1 \pm 0.6 \mathrm{~kg}$; $P<0.01)$.

Mild hypoglycemia was defined as a symptomatic episode or blood glucose less than $3.89 \mathrm{mmol} / \mathrm{L}$, and occurred in $53 \%$ of patients in the exenatide group and $41 \%$ of those in the placebo group $(P<0.05)$. No moderate or severe hypoglycemic episodes were reported. Moderate was defined as $\mathrm{BG}<2.78 \mathrm{mmol} / \mathrm{L}$, and severe episodes required third-party assistance. Withdrawals due to adverse events or gastrointestinal side effects were not presented. 


\section{Liraglutide}

Morrow et al examined the pharmacokinetic (PK) and pharmacodynamic (PD) effects of combining liraglutide with insulin detemir. ${ }^{21}$ This was an open-label study of 32 insulin-naive patients with T2DM over the age of 18 years on stable doses of oral antihyperglycemic agents (metformin with or without other oral agents) for the preceding 2 months. Additional inclusion criteria included the following: BMI $\leq 45 \mathrm{~kg} / \mathrm{m}^{2}, \mathrm{~A} 1 \mathrm{c}$ on or between $7.0 \%$ and $10.0 \%$ for metformin monotherapy, and on or between $7.0 \%$ and $9.5 \%$ for dual therapy. Subjects on dual therapy with an FPG $>13.9 \mathrm{mmol} / \mathrm{L}$ were excluded from the study.

During a 3 week washout period, subjects remained on metformin and other agents were discontinued. On day 1 of the study, all subjects received a single dose of detemir $(0.5$ units $/ \mathrm{kg})$ and proceeded to undergo a 24-hour euglycemic clamp. Subjects were then given liraglutide, which was titrated as follows: $0.6 \mathrm{mg} /$ day for 1 week, $1.2 \mathrm{mg} /$ day for the second week, followed by $1.8 \mathrm{mg}$ daily. A second euglycemic clamp was conducted on day 22 (steady state of liraglutide $1.8 \mathrm{mg}$ ). Liraglutide $1.8 \mathrm{mg} /$ day was continued, and on day 36 subjects were given a single dose of insulin detemir ( 0.5 units $/ \mathrm{kg}$ ) in addition to lirgalutide, and a third euglycemic clamp was performed. Blood glucose levels were maintained at $5.5 \mathrm{mmol} / \mathrm{L} \pm 10 \%$ during the euglycemic clamp studies. PK properties of detemir, liraglutide, and combination of detemir and liraglutide were conducted with 24-hour blood glucose profiles on days 1,22 , and 36 , respectively. On day 36 , PD properties of combination therapy were also assessed.

Detemir PK profiles were not altered by coadministration of liraglutide. The area under the curve (AUC) and maximum concentration $\left(\mathrm{C}_{\max }\right)$ ratios for detemir with concurrent liraglutide vs detemir alone met the predefined no-effect interval of 0.80-1.25 (1.03 [90\% CI 0.97-1.09] and 1.05 [0.98-1.13], respectively). Time to maximum plasma concentration $\left(\mathrm{t}_{\max }\right)$ for detemir was reported as a median of 9.5 hours with and without liraglutide. The $\mathrm{AUC}$ and $\mathrm{C}_{\max }$ ratios for liraglutide alone compared to combination with insulin detemir also indicated no effect (0.97 [0.87-1.08] and 1.03 [0.93-1.13], respectively). The median $t_{\text {max }}$ for liraglutide monotherapy was 11 hours, and 10 hours with the coadministration of detemir. The average AUC glucose infusion rate during the euglycemic clamp studies was $1058 \mathrm{mg} / \mathrm{kg}$ for detemir, $1982 \mathrm{mg} / \mathrm{kg}$ for liraglutide, and $2947 \mathrm{mg} / \mathrm{kg}$ for liraglutide with detemir, suggesting additive glucose-lowering effects with combination therapy.

Two subjects experienced asymptomatic hypoglycemic episodes $(\leq 3.9 \mathrm{mmol} / \mathrm{L})$ during treatment with liraglutide.
There were no adverse events that resulted in subject discontinuation from the study. The most common adverse events $(\geq 10 \%)$ were: abdominal discomfort, diarrhea, nausea, weight decrease, and headache. Gastrointestinal adverse events were mainly reported during liraglutide dose titration. Headaches were mainly reported during clamp studies.

\section{Lixisenatide}

Lixisenatide is an investigational GLP-1RA currently in phase 3 clinical trials. Seino et al conducted a randomized, double-blind, placebo-controlled, parallel group, multicenter, 24-week trial; available as an abstract (Table 1). ${ }^{22}$ This study included Asian participants (from Japan, South Korea, Taiwan, and the Philippines) with T2DM treated with basal insulin with or without a sulfonylurea. A total of 311 subjects were randomized to receive lixisenatide $20 \mathrm{mcg}$ once daily $(n=154)$ or placebo $(n=157)$. The primary end point was change in A1c at the end of the 24 weeks compared to baseline.

A1c reduction was greater in the lixisenatide group than the placebo group (mean \pm SE, $-0.77 \pm 0.14 \mathrm{vs}+0.11 \% \pm 0.13 \%$; between-group difference, $-0.88 \%[-1.12 \%$ to $-0.65 \%]$; $P<0.0001)$. The proportion of patients achieving an A1c of $<7 \%$ and $\leq 6.5 \%$ was both greater in the lixisenatide group compared to the placebo group (35.6\% vs $5.2 \%$ and $17.8 \%$ vs $1.3 \%$, respectively; $P<0.0001)$. Lixisenatide improved FPG levels compared to placebo $(-0.42 \pm 0.31$ vs $0.25 \pm 0.30 \mathrm{mmol} / \mathrm{L}$; between-group difference $-0.67[-1.23$ to $-0.11 \mathrm{mmol} / \mathrm{L}] ; P=0.02$ ), as well as 2 -hour PPG levels $(-7.98 \pm 0.60 \mathrm{vs}-0.14 \pm 0.56 \mathrm{mmol} / \mathrm{L}$; between-group difference, $-7.83 \mathrm{mmol} / \mathrm{L}[-8.89$ to $-6.79 \mathrm{mmol} / \mathrm{L}] ; P<0.0001$ ). Glucose excursions and average 7-point self-monitoring plasma glucose values were also improved with lixisenatide compared to placebo ( $P<0.0001$ for both). Change in weight with lixisenatide was not significantly different from placebo $(-0.38 \pm 0.28$ vs $0.06 \pm 0.27 \mathrm{~kg}$; between-group difference, $-0.43 \mathrm{~kg}$ ( -0.93 to $0.06 \mathrm{~kg}$; $P=0.09)$.

Symptomatic hypoglycemia was reported in $42.9 \%$ of lixisenatide-treated and $23.6 \%$ of placebo-treated patients. Of those not treated with sulfonylureas, symptomatic hypoglycemia rates were $31.8 \%$ in the lixisenatide group and $28.3 \%$ in the placebo group. No cases of severe hypoglycemia were reported. Higher discontinuation due to treatment-emergent adverse events was reported with lixisenatide compared to placebo ( $9.1 \%$ vs $3.2 \%$, respectively), which was mainly attributed to higher rates of nausea $(39.6 \%$ vs $4.5 \%)$ and vomiting (18.2\% vs $1.9 \%)$. 


\section{Discussion}

T2DM is a progressive disease that typically requires the sequential up-titration and addition of antihyperglycemic therapy. Metformin should be prescribed initially for all patients without contraindications. ${ }^{6}$ In most cases, basal insulin is eventually necessary to achieve glycemic control. Unfortunately, approximately $50 \%-70 \%$ of patients fail to achieve A1c goals after one year of titrated basal insulin..$^{23,24}$ For such patients, there are several adjunctive antihyperglycemic therapies to consider. Sulfonylureas and pioglitazone are not recommended in combination with insulin due to increased risk of hypoglycemia and fluid overload, respectively, and additive weight gain for both. Rapid-acting prandial insulin, exenatide, and sitagliptin all target PPG, and thus deserve consideration as add-on therapies to basal insulin.

In deciding among these three options, consideration should be given to glycemic efficacy, effect on cardiovascular risk factors, hypoglycemia, safety, tolerability, patient preference, and cost-effectiveness. ${ }^{6}$ In clinical trials of approximately 6 months' duration, each of these therapies added to basal insulin has been shown to reduce A1c by $0.3 \%$ to $0.7 \%$ as compared to baseline or inactive control. ${ }^{17,19,25-28}$ However, head-to-head studies are needed for accurate comparison. Mendoza and colleagues reported an open-label pre-post study in which 24 patients with T2DM prescribed insulin detemir and insulin aspart (mean dose 40 units aspart daily) were given exenatide twice daily in place of aspart. ${ }^{29} \mathrm{~A} 1 \mathrm{c}$ at baseline and after 6 months were similar $(7.1 \% \pm 0.9 \%$ vs $6.8 \% \pm 0.7 \%$; not significant) but mean plasma glucose and glucose increase above baseline were significantly lower after treatment with exenatide. Exenatide was associated with a substantial reduction in body weight $(-4.7 \mathrm{~kg} \pm 1.0 \mathrm{~kg}$; $P<0.001)$ from baseline. The aforementioned study by Arnolds and colleagues compared the addition of exenatide versus sitagliptin to basal insulin. ${ }^{19} \mathrm{~A} 1 \mathrm{c}$ was reduced $0.6 \%$ with exenatide and $0.3 \%$ with sitagliptin compared to control $(P<0.05$ for exenatide vs control only). Exenatide was associated with weight loss $(-0.9 \mathrm{~kg} ; P<0.05$ vs control $)$ and sitagliptin with weight maintenance, while control was associated with 0.4-kg weight gain. Hypoglycemia occurred more frequently with exenatide compared to sitagliptin (10.1 events/patient-year vs 3.3 events/patient-year). GLP-1RAs are the most expensive of the three options; however, no cost-effectiveness analyses have been completed to compare among these therapies. It is important to consider cardiovascular outcomes in analysis of cost-effectiveness. GLP1RAs are associated with significant decreases in weight, blood pressure, and markers of inflammation as well as improvement in some lipid parameters. ${ }^{30}$ Whether this will translate into improved cardiovascular outcomes is being assessed in several ongoing trials. ${ }^{14}$ Additionally, it is possible that GLP-1RAs may preserve beta-cell function and have cardio- and neuroprotective effects. ${ }^{31}$ Further comparison studies will allow for more evidence-based decision-making among these agents.

The studies reviewed above provide interventional data for the use of GLP-1RAs used in combination with insulin therapy. According to these studies, this combination provides additional A1c lowering when compared to basal insulin control $(0.6 \%-0.88 \%)$, and also resulted in lower basal insulin requirements. Decreases in PPG levels following GLP-1RA administration were consistent across studies, when reported. The improvement in glycemic control did not translate into major/severe hypoglycemic events, as no such episodes were reported. Rates of mild hypoglycemia with GLP-1RAs were either the same or increased when compared to basal insulin control groups. Change in weight was observed in all studies except for the liraglutide PK/PD study (weight was not assessed) and the lixisenatide study conducted by Seino et al whose population was $100 \%$ Asian, with an average baseline BMI of $25.3 \mathrm{~kg} / \mathrm{m}^{2}$; however, these patients were treated with sulfonylureas at baseline and some continued therapy throughout the study. Furthermore, the PK and PD parameters with liraglutide in combination with detemir do not appear to be altered with combination therapy. However, this cannot be extrapolated to other GLP-1RA agents. The most common adverse events reported with the GLP-1RAs were gastrointestinal in nature (nausea, vomiting, and diarrhea) and consistent with previous literature. No cases of pancreatitis were reported during any of these studies.

Use of these agents in clinical practice may warrant adjustments of other diabetes medications. Two of the studies above decreased basal insulin doses prior to the administration of exenatide. One study decreased basal insulin doses by $20 \%$ only in patients with an A1c below $8 \%$ and the other decreased insulin doses by $20 \%$ in all patients regardless of baseline A1c. Combining GLP-1RAs with basal insulin may require decreases in insulin doses in patients at higher risk for hypoglycemia; this should be evaluated on an individual basis. Due to the increased risk of hypoglycemia reported with sulfonylureas, consideration should be given to decreasing the dose or discontinuing these agents. Other oral agents studied above (metformin and pioglitazone) were tolerated with combination therapy and did not require any dosage adjustments. 
Several important limitations of this systematic review should be noted. First, our search was limited to Englishlanguage articles. Second, publication bias of the available literature cannot be excluded. To minimize the risk for potential publication bias, we searched multiple databases, included unpublished data in abstract form and searched reference lists of identified studies. Third, the open-label, crossover pharmacokinetic study conducted by Morrow and colleagues was nonrandomized. In this study, subjects served as their own controls, minimizing the potential for differences in baseline prognoses between treatment sequences; however, the effect of treatment period on outcome measures cannot be systematically assessed in nonrandomized crossover study designs. Finally, only $60 \%$ of included trials by design were blinded.

As previously mentioned, a major limitation of the existing evidence is the lack of long-term comparative effectiveness trials examining the addition of rapid-acting prandial insulin, GLP-1RAs, and DPP-IV inhibitors to basal insulin with or without metformin. The two trials conducted to date were limited by study design and duration of therapy. ${ }^{19,29}$ Future studies comparing the addition of these antihyperglycemic agents to basal insulin with or without metformin are needed. These studies should assess glycemic efficacy, safety, and cardiovascular outcomes and evaluate the cost-effectiveness of these different therapeutic modalities.

\section{Conclusion}

GLP-1RA use in combination with insulin therapy is becoming more common since the FDA and the European Medicines Agency approved the use of exenatide with insulin glargine (with or without metformin and/or a TZD). Adding a GLP-1RA to an existing basal insulin regimen is a reasonable treatment strategy in nonpregnant adult patients with T2DM. This treatment option offers advantages for the treatment of diabetes, such as additional lowering of A1c without a major risk for hypoglycemia, lower basal insulin requirements, decreased PPG levels (with or without FPG decreases), and weight loss, or at the very least, less weight gain. The benefit of adding these agents should be weighed against the gastrointestinal side-effect profile and the cost of these agents. Future studies are needed to assess the long-term benefits of adding GLP-1RAs to insulin therapy compared to other commonly utilized agents.

\section{Disclosure}

The authors report no conflicts of interest in this work.

\section{References}

1. Flegal KM, Carroll MD, Ogden CL, Curtin LR. Prevalence and trends in obesity among US adults, 1999-2008. JAMA. 2010;303: 235-241.

2. Centers for Disease Control and Prevention. National Diabetes Fact Sheet, 2011. Atlanta: US Department of Health and Human Services, Centers for Disease Control and Prevention; 2011.

3. Arbeeny CM. Addressing the unmet medical need for safe and effective weight loss therapies. Obes Res. 2004;12:1191-1196.

4. UK Prospective Diabetes Study (UKPDS) Group. Intensive bloodglucose control with sulphonylureas or insulin compared with conventional treatment and risk of complications in patients with type 2 diabetes (UKPDS 33). Lancet. 1998;352:837-853.

5. UK Prospective Diabetes Study (UKPDS) Group. Effect of intensive blood-glucose control with metformin on complications in overweight patients with type 2 diabetes (UKPDS 34). Lancet. 1998;352: 854-865.

6. Inzucchi SE, Bergenstal RM, Buse JB, et al. Management of hyperglycemia in type 2 diabetes: a patient-centered approach: position statement of the American Diabetes Association (ADA) and the European Association for the Study of Diabetes (EASD). Diabetes Care. Epub April 19, 2012.

7. Nauck MA, Wollschlager D, Werner J, et al. Effects of subcutaneous glucagon-like peptide 1 (GLP-1 [7-36 amide]) in patients with NIDDM. Diabetologia. 1996;39:1546-1553.

8. Larsson H, Holst JJ, Ahren B. Glucagon-like peptide-1 reduces hepatic glucose production indirectly through insulin and glucagon in humans. Acta Physiol Scand. 1997;160:413-422.

9. Flint A, Raben A, Astrup A, Holst JJ. Glucagon-like peptide 1 promotes satiety and suppresses energy intake in humans. J Clin Invest. 1998;101: 515-520.

10. Drucker DJ. Glucagon-like peptides. Diabetes. 1998;47:159-169.

11. Bagger JI, Knop FK, Lund A, Vestergaard H, Holst JJ, Vilsboll T. Impaired regulation of the incretin effect in patients with type 2 diabetes. J Clin Endocrinol Metab. 2011;96:737-745.

12. Shyangdan DS, Royle P, Clar C, Sharma P, Waugh N, Snaith A. Glucagon-like peptide analogues for type 2 diabetes mellitus. Cochrane Database Syst Rev. 2011;10:CD006423.

13. Byetta (exenatide) [package insert]. San Diego: Amylin Pharmaceuticals; 2009.

14. Pinelli NR, Hurren KM. Efficacy and safety of long-acting glucagonlike peptide-1 receptor agonists compared with exenatide twice daily and sitagliptin in type 2 diabetes mellitus: a systematic review and meta-analysis. Ann Pharmacother. 2011;45:850-860.

15. Victoza (liraglutide) [package insert]. Princeton: Novo Nordisk; 2010.

16. Moher D, Liberati A, Tetzlaff J, Altman DG. Preferred reporting items for systematic reviews and meta-analyses: the PRISMA statement. Ann Intern Med. 2009;151:264-269.

17. Buse JB, Bergenstal RM, Glass LC, et al. Use of twice-daily exenatide in basal insulin-treated patients with type 2 diabetes: a randomized, controlled trial. Ann Intern Med. 2011;154:103-112.

18. Riddle MC, Rosenstock J, Gerich J. The treat-to-target trial: randomized addition of glargine or human NPH insulin to oral therapy of type 2 diabetic patients. Diabetes Care. 2003;26:3080-3086.

19. Arnolds S, Dellweg S, Clair J, et al. Further improvement in postprandial glucose control with addition of exenatide or sitagliptin to combination therapy with insulin glargine and metformin: a proof-of-concept study. Diabetes Care. 2010;33:1509-1515.

20. Riddle M, Ahmann A, Basu A, Aroda V, Ratner R. Metformin+ exenatide+basal insulin vs metformin+placebo+basal insulin: reaching A1c $<6.5 \%$ without weight-gain or serious hypoglycemia. Diabetes. 2010;59(Suppl 1):Abstract 18-LB.

21. Morrow L, Hompesch M, Guthrie H, Chang D, Chatterjee DJ. Co-administration of liraglutide with insulin detemir demonstrates additive pharmacodynamic effects with no pharmacokinetic interaction. Diabetes Obes Metab. 2011;13:75-80. 
22. Seino Y, Min K, Niemoller E, Takami A. Lixisenatide significantly improves glycemic control in Asian patients with T2DM insufficiently controlled on basal insulin +/- SU. Diabetes. 2011;60(Suppl 1): Abstract 278-OR.

23. Holman RR, Thorne KI, Farmer AJ, et al. Addition of biphasic, prandial, or basal insulin to oral therapy in type 2 diabetes. N Engl J Med. 2007; 357:1716-1730.

24. Rosenstock J, Davies M, Home PD, Larsen J, Koenen C, Schernthaner G. A randomised, 52-week, treat-to-target trial comparing insulin detemir with insulin glargine when administered as add-on to glucose-lowering drugs in insulin-naive people with type 2 diabetes. Diabetologia. 2008;51:408-416.

25. Apidra (insulin glulisine [rDNA origin] injection) [package insert]. Bridgewater: Sanofi-Aventis US; 2009.

26. Humalog (insuln lispro injection) [package insert]. Indianapolis: Lilly USA; 2011.
27. Novolog (insulin aspart [rDNA origin] injection) [package insert]. Princeton: Novo Nordisk; 2009.

28. Vilsboll T, Rosenstock J, Yki-Jarvinen H, et al. Efficacy and safety of sitagliptin when added to insulin therapy in patients with type 2 diabetes. Diabetes Obes Metab. 2010;12:167-177.

29. Mendoza CA, Mathew M, Chen J, et al. Exenatide promotes weight loss and improves insulin secretion, subclinical inflammation and hepatic steatosis when replaced for pre-meal insulin in T2DM patients. Diabetes. 2009;58(Supp 1):Abstract 507.

30. Davidson MH. Cardiovascular effects of glucagonlike peptide-1 agonists. Am J Cardiol. 2011;108:33B-41B.

31. Drucker DJ. The biology of incretin hormones. Cell Metab. 2006;3: 153-165.

Diabetes, Metabolic Syndrome and Obesity: Targets and Therapy

Dovepress

\section{Publish your work in this journal}

Diabetes, Metabolic Syndrome and Obesity: Targets and Therapy is an international, peer-reviewed open-access journal committed to the rapid publication of the latest laboratory and clinical findings in the fields of diabetes, metabolic syndrome and obesity research. Original research, review, case reports, hypothesis formation, expert

opinion and commentaries are all considered for publication. The manuscript management system is completely online and includes a very quick and fair peer-review system, which is all easy to use. Visit http://www.dovepress.com/testimonials.php to read real quotes from published authors.

Submit your manuscript here: http://www.dovepress.com/diabetes-metabolic-syndrome-and-obesity-targets-and-therapy-journal 